\title{
A Machine Learning Classifier for Fast Radio Burst Detection at the VLBA
}

Kiri L. Wagstaff ${ }^{1}$, Benyang Tang ${ }^{1}$, David R. Thompson ${ }^{1}$, Shakeh Khudikyan ${ }^{1}$, Jane Wyngaard $^{1}$, Adam T. Deller ${ }^{2}$, Divya Palaniswamy ${ }^{3,4}$, Steven J. Tingay ${ }^{3,5}$, and Randall B. Wayth ${ }^{3}$

kiri.1.wagstaff@jpl.nasa.gov

\begin{abstract}
Time domain radio astronomy observing campaigns frequently generate large volumes of data. Our goal is to develop automated methods that can identify events of interest buried within the larger data stream. The V-FASTR fast transient system was designed to detect rare fast radio bursts (FRBs) within data collected by the Very Long Baseline Array. The resulting event candidates constitute a significant burden in terms of subsequent human reviewing time. We have trained and deployed a machine learning classifier that marks each candidate detection as a pulse from a known pulsar, an artifact due to radio frequency interference, or a potential new discovery. The classifier maintains high reliability by restricting its predictions to those with at least $90 \%$ confidence. We have also implemented several efficiency and usability improvements to the V-FASTR web-based candidate review system. Overall, we found that time spent reviewing decreased and the fraction of interesting candidates increased. The classifier now classifies (and therefore filters) $80-90 \%$ of the candidates, with an accuracy greater than $98 \%$, leaving only the 10-20\% most promising candidates to be reviewed by humans.
\end{abstract}

Subject headings: methods: data analysis - Astronomical Instrumentation, Methods and Techniques

\section{Introduction}

The Very Long Baseline Array (VLBA) consists of 10 widely separated radio antennas. Their locations range from Mauna Kea, Hawaii, to St. Croix in the Virgin Islands, yielding baselines of

\footnotetext{
${ }^{1}$ Jet Propulsion Laboratory, California Institute of Technology, 4800 Oak Grove Drive, Pasadena, CA 91109, USA

${ }^{2}$ ASTRON, the Netherlands Institute for Radio Astronomy, Oude Hoogeveensedijk 4, 7991 PD Dwingeloo, The Netherlands

${ }^{3}$ ICRAR, Curtin University, Bentley, WA 6845, Australia

${ }^{4}$ University of Nevada, 4505 S. Maryland Parkway, Las Vegas, NV 89154, USA

${ }^{5}$ ARC Centre of Excellence for All-Sky Astrophysics (CAASTRO)
} 
up to $8000 \mathrm{~km}$. Data is collected individually by each $25 \mathrm{~m}$ antenna and then sent to the correlator in Socorro, NM. Excellent time and angular resolution enable precise detection and localization of coincident signals observed by multiple antennas.

Fast radio bursts (FRBs) (Lorimer et al. 2007; Thornton et al.|2013; Burke-Spolaor and Bannister 2014) are radio phenomena of particular interest. These are non-repeating, short-duration (millisecond to sub-millisecond), broad-band radio pulses that are observed on Earth with a frequencydependent time of arrival. The amount of dispersion, or the delay between the arrival of the signal at the highest and lowest frequencies observed, is dependent on the path length of the signal through ionized plasma (and its density) between the source and the observer. Highly dispersed transient events are of particular interest since they may have an extragalactic origin. Potential sources of these events include gamma-ray bursts (GRBs) (Zhang 2014), supra-massive neutron stars (Falcke and Rezzolla 2014), binary neutron star mergers (Totani 2013), binary white dwarf mergers (Kashiyama et al. 2013), flaring stars (Loeb et al. 2014), and many more.

In addition to astronomical signals, local (Earth-based) sources of interference can generate intrinsically dispersed signals that in some ways resemble FRBs. One prominent example was the discovery of the so-called "Perytons" (Burke-Spolaor et al. 2011) that ultimately were determined to have been generated by a microwave oven near the Parkes radio telescope (Petroff et al. 2015b). Even within sources categorized as astronomical FRBs, there is considerable variety: the prototypical Lorimer Burst (Lorimer et al. 2007) is far brighter than any of the subsequently discovered bursts, for instance. It would significantly improve our understanding of these events to localize an incoming pulse through wide baseline interferometry. However, this would require re-analyzing the data after a pulse is identified, demanding archival storage of an infeasible quantity of raw data.

To better understand the variety and incidence of FRBs, we seek to detect and catalog as many such events as possible. The ideal transient detection system would be able to detect FRBs across a wide range of frequencies, produce high time-resolution data, discriminate against RFI, have good sky coverage, and localize the burst on the sky with enough angular resolution to identify the origin of the burst. The VLBA is well suited to such a task, and the V-FASTR (VLBA Fast Transient) system (Thompson et al. 2011; Wayth et al. 2011) was created to search data collected by the VLBA for FRBs.

\subsection{The V-FASTR System for FRB Detection}

Searches for transients in the image domain are usually confined to longer-duration events ( $\sim$ seconds or longer), due to the extreme computational complexity of searching for short and dispersed signals such as single pulses from radio pulsars or FRBs. An overview of current and future image plane transient searches is given by Fender et al. (2015). Searches for fast radio transients use short integration times (millisecond or shorter) to preserve sensitivity, but they are usually carried out on large single dishes with narrow fields of view (FOV), poor angular resolution, 
and high sensitivity to RFI. However, we note that some pilot studies using imaging to search for fast radio transients have been carried out (Law et al. 2015).

The VLBA provides a smaller FOV $\left(0.27 \mathrm{deg}^{2}\right)$ than other similar experiments (Wayth et al. 2011) with a sensitivity of $0.3 \mathrm{Jy}(1.4 \mathrm{GHz})$. As a set of distributed antennas, however, the VLBA offers a distinct advantage in RFI rejection because signals that are not observed by multiple antennas can be automatically filtered out. The VLBA's long baseline (up to $8000 \mathrm{~km}$ ) enables extremely good localization of any detected sources (within a few milliarcseconds) which is invaluable for interpreting and following up on any detections.

Further, the VLBA's flexible DiFX software correlator (Deller et al. 2011) enables the generation of short-integration spectrometer data for each antenna at minimal additional cost as part of the $\mathrm{F}$ stage, and because the time and frequency resolution properties of the spectrometer data are configurable, it is possible to customize the processing. A 1-ms time resolution for observing frequencies around $1.4 \mathrm{GHz}$ was chosen for V-FASTR as a compromise between signal detectability and data volume or computational effort.

V-FASTR was designed to operate commensally at the VLBA, meaning that it passively analyzes all data collected by the array in support of a variety of imaging campaigns. This enables the potential for FRB detections even in campaigns with other primary scientific goals. In 2014, V-FASTR was also granted 700 hours of its own observing time to conduct a more systematic scan and reduce the sampling bias induced by observing only those sky locations selected by investigators for other purposes.

V-FASTR employs a highly efficient, real-time candidate detection system that processes incoming VLBA data and saves out information about possible FRBs (Thompson et al. 2011; Wayth et al. 2011). FRB candidates are defined as strong signals that are correlated across multiple antennas. V-FASTR balances sensitivity with robustness by adaptively deciding which antennas to use based on the current noise environment. VFASTR's real-time "triage" operation permits analysis of much larger data volumes than would be possible for offline processing. While it analyzes a majority of VLBA data, it only preserves the tiny fraction of voltage data associated with candidate events. Later, those candidates are reviewed by experts to determine whether they originate from a known source (e.g., pulsar), an artifact or radio frequency interference (RFI), or a truly novel source. The review process is enabled by a web-based classification and review system that provides a human-friendly interface for reviewing the tens to hundreds of candidates detected per day (Hart et al. 2014).

To date, no new FRBs have been detected, but the time spent observing at a range of frequencies and pointing locations has informed the determination of upper limits on the expected rate of such events (Wayth et al. 2012; Trott et al. 2013). Blind detections of many known pulsars (Thompson et al. 2013) have validated the system's sensitivity. V-FASTR serves as a pathfinder experiment to illustrate how commensal science can be done, both for added value in current science campaigns and as a way to scale up to the unprecedented data volumes anticipated for the Square Kilometre 
Array (Macquart et al. 2014) and other future instruments.

\subsection{Contributions}

In this paper, we describe two new advances to the V-FASTR review system that were designed to reduce the human effort required to evaluate the V-FASTR candidates. First, we developed a machine learning classifier that automatically predicts the correct classification of $80-90 \%$ of the V-FASTR candidates, tagging them as created by known pulsars or RFI. By pre-classifying a large proportion of uninteresting (to this experiment) candidates, the classifier enables human reviewers to focus their time on the remaining potentially interesting candidates. As each such candidate is considered and tagged with its appropriate class by human reviewers, the classifier re-trains with this new information and continually improves its knowledge of radio transient characteristics.

Second, we implemented several improvements to the V-FASTR web-based candidate review portal. This system is vital to support the geographically distributed team of reviewers. The interface enables the fast compilation of summary statistics about the candidates that have been detected and, as noted above, it also enables continual improvement for the machine classifier in the form of new feedback from the reviewers. We have implemented (1) efficiency improvements that greatly increased the responsiveness of the system, (2) user interface improvements identified by a user study, (3) and user authentication to improve usability (by providing a customized review list for each user) and enable per-user activity tracking.

Taken together, these advances contribute to a solution to the data volume challenge involved in VLBA data processing and radio transient detection. In addition to rapid data triage by the real-time detection system, it is vital to minimize the human effort required to review the candidate events. The V-FASTR classifier provides a necessary initial step that sets aside candidates that are known to be uninteresting and enables reviewers to devote their time to the review of the remaining candidates. The improved interactive web interface serves the dual purposes of visualizing the candidates and collecting human evaluations of each one. This solution is significant given the anticipated arrival of instruments such as the Square Kilometre Array and its predecessors, which promise to increase the potential observational parameter space for future radio astronomy surveys by orders of magnitude (Dewdney et al. 2009). Going forward, V-FASTR will continue to search for, classify, and send alerts for promising candidates, potentially leading to new discoveries.

\section{Background and Related Work}

\subsection{Radio Transient Detection Studies}

A number of other studies have detected radio transients using instruments other than the VLBA. Data collected by the Parkes radio telescope has been studied extensively following the 


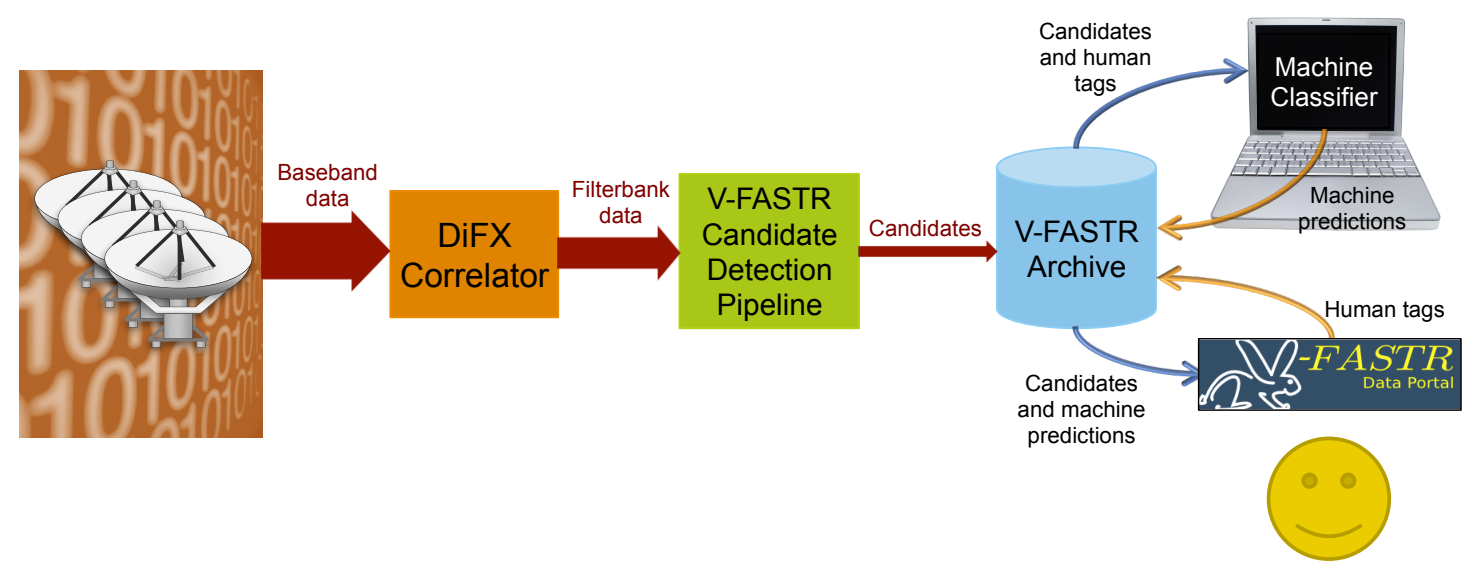

Fig. 1.- The V-FASTR system operates commensally with regular VLBA data collection and correlation. Data is transferred from the antennas to the DiFX software correlator (Deller et al. 2011). Filterbank data output by the spectrometers is analyzed by the real-time candidate detection system (Thompson et al. 2011), and those candidates are stored in the V-FASTR archive. Data volume is reduced at each step. The candidates are shown to human reviewers via the web portal. Candidates tagged by reviewers are used to re-train the classifier, which in turn generates predictions that are displayed to reviewers to aid in their review process.

detection of the Lorimer burst (Lorimer et al. 2007). In an archival survey of Parkes data, Keane et al. (2012) found an FRB that was observed (but unnoticed) in 2001 and proposed that its source could be a radio-emitting magnetar. Thornton et al. (2013) detected four FRBs in newly collected Parkes data since 2010, Burke-Spolaor and Bannister (2014) reported another FRB in data from 2001, and Ravi et al. (2015) and Petroff et al. (2015a) each detected one new FRB during real-time observing in 2013 and 2014, respectively.

Spitler et al. (2014) reported the first FRB detection on an instrument other than the Parkes radio telescope (the Arecibo L-Band Feed Array or ALFA). Siemion et al. (2012) searched 450 hours of data from the Allen Telescope Array (ATA) but did not find any new transients. Masui et al. (2015) found an FRB in archived data from the Green Bank Hydrogen Intensity Mapping survey.

\subsection{The V-FASTR Radio Transient Detection and Review System}

Figure 1 outlines the complete V-FASTR system. Each VLBA observation can employ up to ten 25-m antennas for simultaneous observations from different geographic locations. Once the observation is complete, hard drives containing the raw (baseband) data are shipped from each antenna to the Array Operations Center of the National Radio Astronomy Observatory in Socorro, NM, for correlation and analysis. The V-FASTR event detection pipeline runs in real time 
in parallel with the DiFX correlator. The correlator generates filterbank data that captures the observed signal intensity at each antenna for a range of frequency bins as a function of time. VFASTR employs a robust, adaptive summing technique to detect candidate events in the filterbank data (Thompson et al. 2011). The data associated with each candidate (including, for the top few candidates per hour, raw voltage data that would enable localization to the milliarcsecond level) is saved to disk, and figures are generated to display the data for human review. The rest of the data is discarded, and the hard drives are erased once correlation completes to enable their re-use for future observations. Meta-data associated with each detection, including its timestamp, the array pointing sky coordinates (right ascension and declination), the signal strength, and the dispersion measure, are also saved to aid in the candidate review process.

In order to ensure that no interesting event is missed, the real-time system uses a lenient threshold that also admits some non-FRB events. Candidates consist of pulsar pulses, spurious correlated radio frequency interference (RFI), and other potentially unknown phenomena. The number of candidates generated by V-FASTR each day ranges from zero to tens to hundreds to thousands, depending on the observational target and environmental (temperature and RFI) conditions. These candidates are highly diverse, and no simple rule can easily recognize or anticipate all cases. Consequently, human review is the safest way to filter them further without missing an interesting event.

We developed a candidate event classifier to reduce the reviewing burden by automatically tagging events that can be confidently classified as pulses from a known pulsar or as RFI (artifacts). The remaining candidates consist of pulses without a known origin or explanation; these are the candidates that most require human assessment and that have the highest chance of containing a new discovery.

The V-FASTR review process relies on a web-based review portal that offers reviewers worldwide access to the events for review and evaluation (Hart et al. 2014). Reviewers, interested PIs, and the public are able to search and browse the resulting classified candidates 1 . Human review decisions are also used to update and re-train the machine classifier, so that the system automatically and transparently improves over time.

In this paper, we first present the V-FASTR candidate classifier and describe how it is trained and evaluated (Section 3). Next, we describe the web-based review portal and our recent improvements and advances (Section 4). Section 5 shares the results of the classifier's use in the real-time system and web portal. Finally, Section 6 describes the system's current use and discusses how the V-FASTR approach might be used advantageously elsewhere in the future.

${ }^{1}$ http://curta.pawsey.org.au/ 


\section{V-FASTR Candidate Classifier for Radio Transients}

The V-FASTR candidate classifier analyzes each candidate that is detected by the real-time system. It employs a trained random forest classifier (Breiman 2001) to predict the class for each new candidate, then consults a database of known pulsars to further refine its predictions. Predictions that are sufficiently confident are added to the meta-data associated with the candidate and used to reduce the number of candidates that require human review.

\subsection{V-FASTR Classifier Features}

The V-FASTR detection system identifies candidates by de-dispersing the radio frequency data from each observing antenna and computing a robust sum across antennas to identify correlated events (Thompson et al. 2011). Filterbank data for each candidate is saved out to disk for further analysis. Figure 2 shows an example of a candidate event detected by V-FASTR, in which signal strength is shown as a function of frequency ( $\mathrm{y}$ axis) and time (x axis). Vertical red lines indicate the start and end of the detected event. The saved data includes observations for a buffer period before and after the event to provide context for the observing conditions at the time that the event occurred.

For each candidate, the system constructs a feature vector that captures key information needed to classify it. The features were chosen based on years of manual review experience with the types of artifacts and effects the data exhibited during that period. They leverage general image statistics of relevant regions in the data stream before, during, and after a candidate event. The ten features are as follows:

1. The minimum observing frequency (in Hz). This is important because some bands are more prone to RFI than others.

2. The estimated dispersion measure (DM) (in pc/cc). This feature helps exclude non-astrophysical events.

\section{Sum across all antennas}

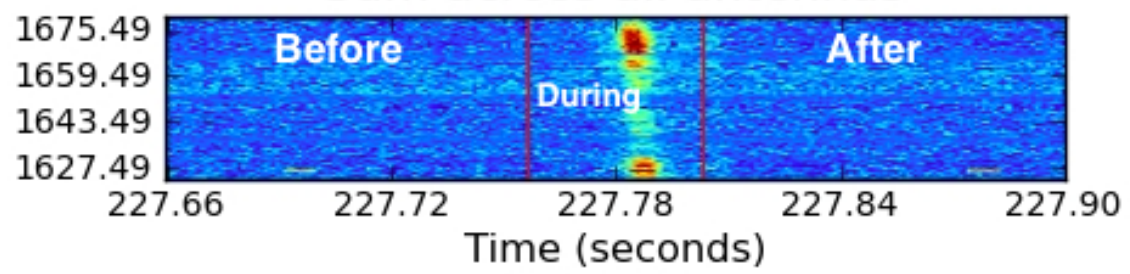

Fig. 2.- Example V-FASTR candidate with signal strength as a function of frequency (in $\mathrm{MHz}$ ) and time. Vertical red lines indicate the start and end of the event. "Before", "during", and "after" regions are used to calculate descriptive features. 
3. The signal to noise ratio (SNR) of the detection. This feature can influence the statistics of many other characteristics, so it is important to consider in the aggregate decision.

4. Max asymmetry: The maximum difference (across frequency channels) in observed intensity before the event versus after the event (see Figure 2). This attribute is often extreme for narrow-band transient RFI.

5. Mean asymmetry: The mean difference (across frequency channels) in observed intensity before the event versus after the event. This feature helps to recognize system state changes which can cause step-function changes in the signal level.

6. Max outlierness: The maximum difference (across frequency channels) in observed intensity during the event versus the concatenation of observations before and after the event. This helps to recognize unstable interference conditions over large numbers of antennas.

7. Mean outlierness: The mean difference (across frequency channels) in observed intensity during the event versus before and after the event.

8. Max zeros: The maximum ratio (across frequency channels) of signal dropouts during the event versus before the event. Network dropouts occasionally occur during correlation, and they can create confusing null data segments in the time series. These segments appear as simultaneous signals across all antennas, so it is important to make an explicit provision to handle them.

9. Mean zeros: The mean ratio (across frequency channels) of signal dropouts during the event versus before the event.

10. The log ratio of covariance across antennas during the event versus before and after the event.

\subsection{V-FASTR Classification Hierarchy: Pulsars, Artifacts, and Good Candidates}

The V-FASTR classifier adopts a two-stage hierarchical approach to classifying candidates. First, a random forest classifier is trained to predict the probability that a candidate is a "pulse" or an "artifact." If the most probable class does not have a posterior probability of at least 0.9 , the classifier abstains (predicts "none"; see the top level of Figure 3).

Candidates classified as "pulse" are further refined by consulting the ATNF Pulsar Catalogue (Manchester et al. 2005), as shown in the bottom level of Figure 3. If the array pointing center is sufficiently close to a known pulsar, and the estimated DM is within $50 \mathrm{pc} / \mathrm{cc}$ of the known DM for that pulsar, then the system changes "pulse" to "pulsar." If not, the system changes "pulse" to "good candidate". These are the candidates that most merit human review, since they have the characteristics of a pulsar pulse (or FRB) but do not correspond to a known source. We define a "sufficiently close" pointing center as one that is within 2 times the full-width half-maximum 


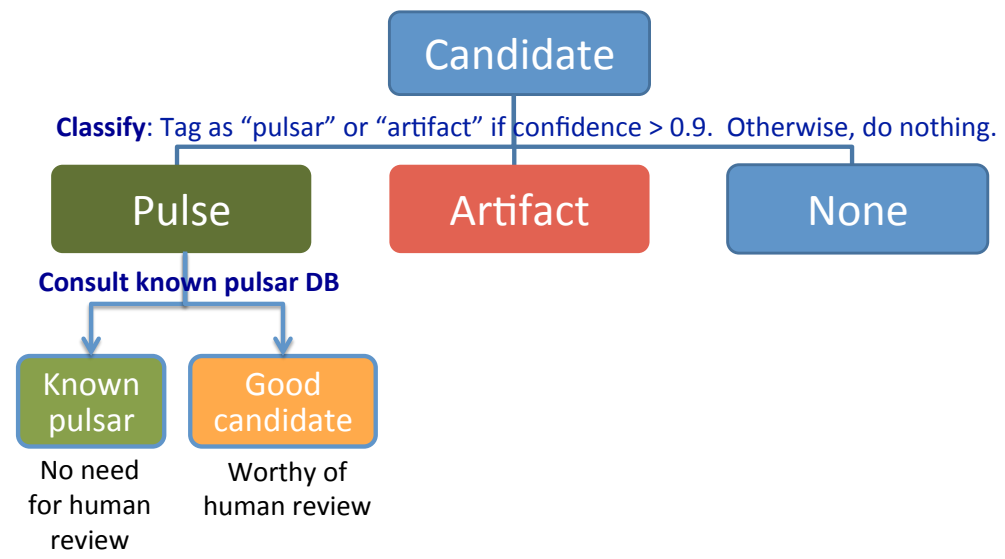

Fig. 3.- Hierarchical V-FASTR candidate classifier structure.

(FWHM) beamwidth of the VLBA configuration when the candidate was detected. FWHM is $1.22 * \lambda / D$, where $\lambda=3.0 \times 10^{8} / f, f$ is the lowest observing frequency, and $D$ is the antenna diameter $(25 \mathrm{~m})$. This two-stage approach to classification reduces the number of pulsar database lookups greatly without increasing the complexity of the classifier.

The benefit of the classifier is in reducing the number of candidates that require human review. Given sufficiently reliable classifier predictions, reviewers can prioritize candidates in this order: "good candidate", unclassified candidates (which could represent a new phenomenon), "pulsar", and "artifact." Therefore, the most promising candidates will be examined first.

\subsection{V-FASTR Classifier Evaluation}

Relying on the classifier to tag, sort, and filter candidates requires that it first demonstrate sufficiently reliable performance. We evaluated performance by collecting 7,649 candidates that were tagged by reviewers as "Pulsar" or "Artifact" and conducting 10-fold cross-validation to assess the classifier's ability to generalize from the training data. We divided the data set into 10 equally sized "folds" and then repeatedly trained a classifier on nine folds and evaluated its performance on the held-out tenth. After doing this 10 times, we had obtained held-out predictions for all of the labeled data and could calculate performance in this simulation of prediction on new data. The classifier had an overall accuracy (agreement with reviewer tags) of $95.8 \%$.

A breakdown of artifact and pulsar classifications on the labeled data set is shown in the confusion matrix in Table 1. For example, the classifier correctly classified 4,433 pulsar candidates. There were 124 false detections and 199 missed detections, yielding a false positive rate of 0.03 and a false negative rate of 0.06 .

Our goal was a classifier with no more than a $5 \%$ (i.e., 0.05 ) false positive rate. The classifier 
Table 1: Confusion matrix showing the number of candidates classified as "Artifact" or "Pulsar" by the classifier (rows) compared to their true class labels (columns). Overall accuracy was 95.8\%.

\begin{tabular}{|l|r|r|r|}
\hline & \multicolumn{2}{|c|}{ True class } & \\
& Artifact & Pulsar & Total \\
\hline Artifact & 2893 & 199 & 3092 \\
Pulsar & 124 & 4433 & 4557 \\
\hline Total & 3017 & 4632 & 7649 \\
\hline
\end{tabular}

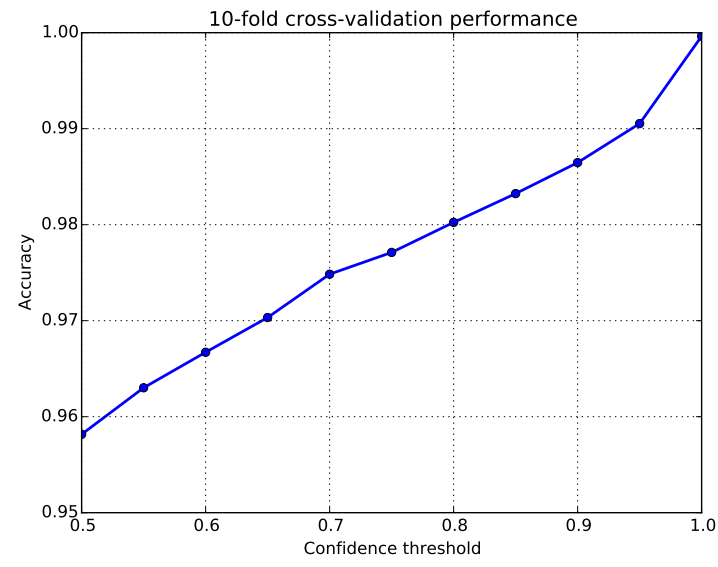

(a) Cross-validated accuracy

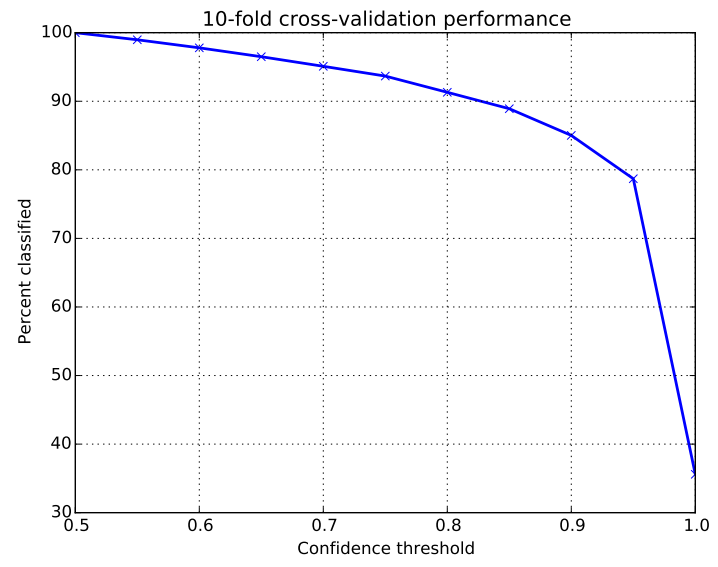

(b) Percent of candidates classified

Fig. 4.- V-FASTR candidate classifier performance as a function of confidence threshold $\tau$, using 10-fold cross-validation.

exceeded this requirement. However, we wanted to improve (reduce) the false negative (miss) rate as well. If we impose a confidence threshold $\tau$, the classifier only generates predictions if they have a posterior confidence $\geq \tau$. By default, $\tau=0.5$ for this two-class classifier, which means that all predictions are used, since at least one class has a posterior probability $>=0.5$. Employing a higher value for $\tau$ increases accuracy (see Figure 4(a)) but reduces the number of predictions made (see Figure 4(b)). For example, the rightmost data point on both plots shows that the classifier achieved 100\% accuracy on its top 36\% most confident predictions. For a confidence threshold of 0.9 , the classifier achieved $98.6 \%$ accuracy while classifying $79 \%$ of the candidates. This is $3.0 \%$ higher than the original performance.

The new confusion matrix is shown in Table 2, In comparison to the original performance obtained when making predictions for all candidates (Table 1), the false positive rate decreased greatly to 0.004 , and the false negative rate halved to 0.03 . We therefore decided to proceed with a confidence threshold of 0.9 in the operational system. 
Table 2: Confusion matrix given a confidence threshold of 0.9. Overall accuracy was 98.6\%. Compare to Table 1 .

\begin{tabular}{|l|r|r|r|}
\hline & \multicolumn{2}{|c|}{ True class } & \\
& Artifact & Pulsar & Total \\
\hline Artifact & 2436 & 73 & 2509 \\
Pulsar & 16 & 4007 & 4023 \\
\hline Total & 2452 & 4080 & 6532 \\
\hline
\end{tabular}

\section{Candidate Review Pipeline and Collaborative Web Portal}

In this section, we describe the candidate review pipeline and recent improvements to the collaborative web-based review system that was originally described by Hart et al. (2014). This system consists of two major components: (1) a metadata pipeline responsible for the capture, transfer, and storage of candidate metadata annotations; and (2) a collaborative web portal which provides analysts with a convenient, context-rich environment for efficiently classifying candidate events.

\subsection{Candidate Metadata Pipeline}

The candidate review system implementation heavily leverages open source software such as Apache OODT $2^{2}$ for managing the metadata and the Apache Solr ${ }^{3}$ fast-response search server. Apache OODT is an information management and processing framework from the Apache Software Foundation recognized for data archiving, metadata extraction, cataloging, querying, and product retrieval. Apache Solr is a widely used open source search platform that provides rapid querying and facet-based search.

We employ the Catalog and Archive Service (CAS) Crawling Framework (Mattmann et al. 2013 ) to run at predefined intervals and automatically detect new candidate products, which triggers the extraction and storage of the metadata in Solr using the OODT File Manager (Mattmann et al. 2013). This metadata includes information about the associated VLBA job's observing parameters (which frequencies and antennas were used and where the array was pointed), the date and time at which the candidate began, the duration of the candidate, the estimated dispersion measure, and more.

$\sqrt[3]{\text { https : //oodt.apache.org/ }}$
$3_{\text {http: //lucene.apache.org/solr/ }}$ 


\subsection{Reviewer Web Portal Interface and Interaction}

The V-FASTR real-time candidate detection system generates key products needed for the review and manual classification of new candidates. These include (1) a "waterfall" plot that shows signal strength as a function of observing frequency and time, at each active antenna, and (2) the de-dispersed time series for each active antenna, using the DM value estimated by the candidate detection system.

The collaborative candidate review web portal provides the geographically distributed VFASTR science team with the ability to quickly examine candidates as they are detected. Figure 5 shows the reviewer interface to the web portal display for an example candidate event. The webpage shows the waterfall plots (left) and de-dispersed time series (right). Vertical red lines indicate the start and end of the event. The portal also reports nearby pulsars (top) as an aid to reviewers, who then have the option of tagging the event using the checkboxes at the bottom.

Reviewers can select from a predefined list of "tags" to specify the candidate's classification (see bottom of Figure 5). The V-FASTR classifier's prediction is shown in a blue highlighted box, along with its posterior confidence. If the confidence is less than 0.9 , the classifier's prediction is not stored, used, or displayed. Reviewers can agree with the classifier, select another tag to correct the classifier, or do nothing. Any new tags created by reviewers are stored in the Solr database. When all of the candidates for a given job have been reviewed, the job is archived. The associated baseband and filterbank data are deleted, except for the filterbank data associated with detected candidates and the baseband data for any candidates marked as "save" by the reviewer (not shown in Figure 5].

Once a day, the V-FASTR classifier checks for new reviewer tags. If any are present, the classifier re-trains on all labeled data. The updated classifier then generates new predictions for all candidates in the database and saves all predictions with confidence greater than 0.9 . In this way, the classifier quickly adapts to any corrections or new information provided by the reviewers. The web portal always displays the latest classifier predictions. This process is illustrated on the right side of Figure 1 .

\subsection{New Review Portal Features}

We have added several new features and capabilities to the V-FASTR web portal. First, we greatly improved its speed and responsiveness by upgrading the system architecture to Solr version 4.5.1, which allows the portal to read and write directly to the Solr database. This improved the speed with which new meta-data, such as new tags created by reviewers, is stored.

Second, we redesigned the portal entry page with a more functional and user-friendly interface (see Figure 6). This page displays a list of the latest jobs that clearly distinguishes those that have been reviewed (green checked box) from those that need to be reviewed (blue unchecked box). 


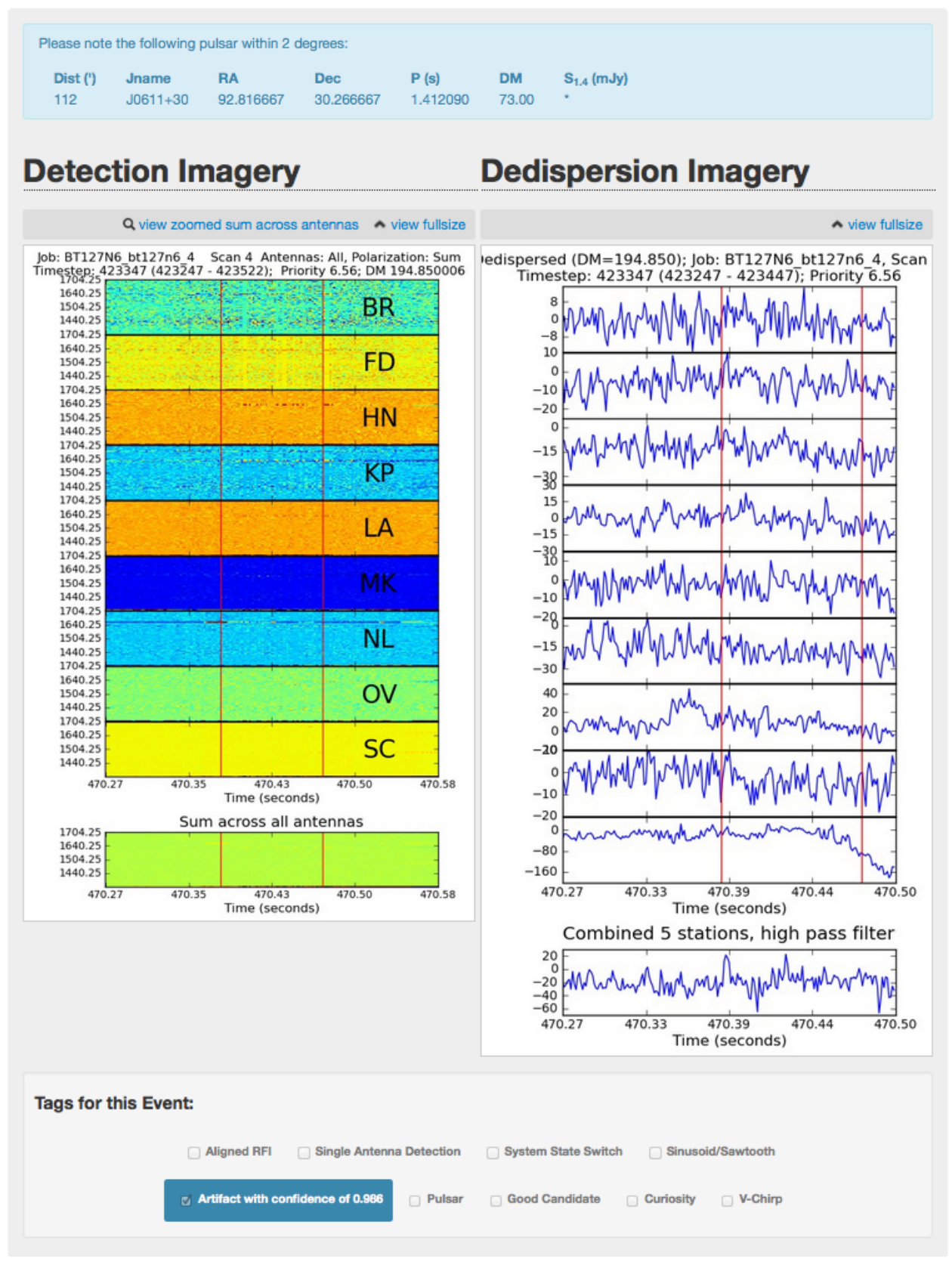

Fig. 5.- Web portal display for a V-FASTR candidate, including waterfall plots (left), de-dispersed time series (right), and nearby pulsars (top). A list of nearby pulsars appears at the top of the page. Reviewers can tag the candidate using the checkboxes at the bottom. The V-FASTR classifier's prediction is shown in a blue highlighted box. 


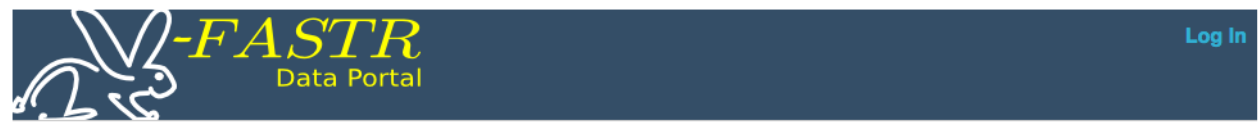

\section{V-FASTR Data Portal $\quad$ Q Search V-FASTR Detections}

Latest Detections 55 Unreviewed jobs are at the top of the list
G00064_04, Dec. 29, 2013, Reviewer: Walid
More Information (15 scans)
G173_11, Oct. 28, 2013, Reviewer: David
More Information (2 scans)
ungatedm5_1, May, 16, 2013, Reviewer: Divya
More Information (5 scans)
gatedk5_2, May. 10, 2013, Reviewer: Sarah
More Information (4 scans)
$\begin{aligned} & \text { gatedk4_3, Mar. 21, 2013, Reviewer: Divya } \\ & \text { More Information (3 scans) }\end{aligned}$ events
BT12708_bt127o8_2, Sep. 12, 2015, Reviewer: Daivd
More Information (2 scans)

Fig. 6.- The V-FASTR web portal interface showing all unreviewed jobs, followed by reviewed jobs, in reverse chronological order.

Unreviewed jobs appear first on the list. Previously, jobs were sorted only by date, and reviewers sometimes had to click through several pages to find the first unreviewed job. Jobs are paginated with 25 jobs per page; Figure 6 shows page 6 of the results to focus on the boundary between the 55 unreviewed jobs and the beginning of the reviewed jobs.

Each job has an assigned reviewer, but any reviewer can review any job, so that the team can accommodate periods when a reviewer is sick, on travel, or otherwise unable to review his or her assigned jobs. Although any reviewer can review any job, it is important that there is a nominal assignment of responsibility to ensure that no candidates fall through the cracks. Each job also has an associated button that indicates the number of candidate events that were found within the job, as a preview of the number of candidates that need to be reviewed.

Third, we added an authentication component so that reviewers can access a customized list of jobs that contains only those jobs for which they are the assigned reviewer. For example, Figure 7 shows the custom review list that reviewer Randall would see. Only jobs assigned to Randall are shown, allowing him to quickly process his jobs. If time permits, he can then click "All Detections" to switch to the full list of all jobs and then browse, or review, any jobs that are available. User authentication now allows us to record the author of each tag. This meta-data allows us to generate statistics about per-reviewer activity. 


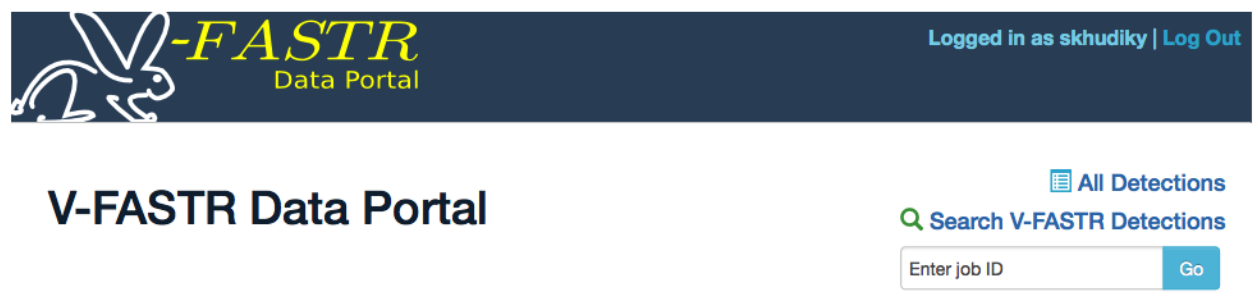

Randall's Detections 5 Unreviewed jobs are at the top of the list

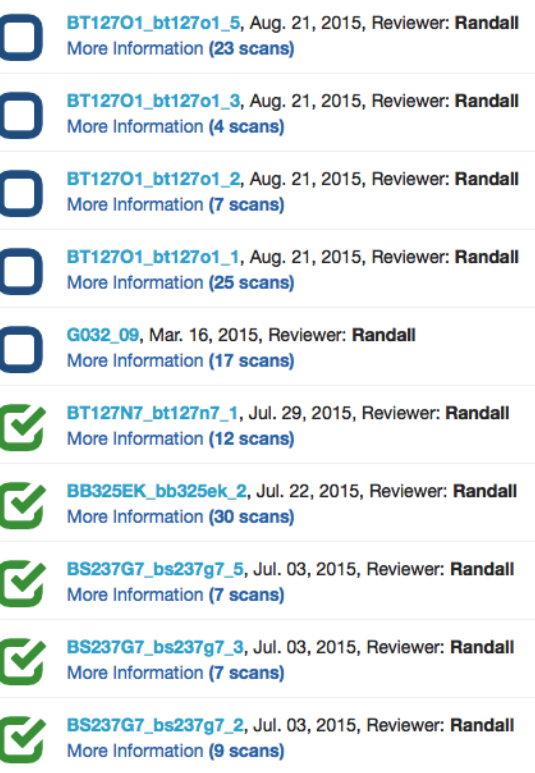

O Inspect 53 events

Inspect 9 events

Inspect 22 events

Inspect 44 events

Inspect 4 events

Inspect 38 events

Inspect 86 events

9 Inspect 10 events

9 Inspect 6 events

9 Inspect 4 events

Fig. 7.- The V-FASTR review portal for a particular reviewer, after logging in. Each reviewer is shown only those jobs assigned to to him or her (compare to Figure 6). Access to the full list of all jobs is available from the "All Detections" link so that reviewers can assist other reviewers with their assigned jobs.

The addition of authentication allowed us to open up the V-FASTR web portal to the public. Only logged-in users are allowed to tag candidates or review jobs, but anyone can browse the V-FASTR jobs and candidates in a read-only mode. This allows other VLBA science PIs and the interested public to see what V-FASTR has detected. Each candidate has its own URL, so reviewers and other users can easily share candidates of interest and discuss them collaboratively. This feature has been utilized by other (non-V-FASTR) VLBA users who are interested in time domain data to inspect potential fast transient sources associated with their observations (e.g., K. Bannister, VLBA project code BB325).

Fourth, we modified how the candidates are shown within each job's review page. Previously, candidates were sorted by the time at which they occurred. We have now integrated the classifier's predicted tags into the portal display so that candidates are shown in the following order: "good 
candidate", unclassifiable, and "pulsar." Candidates classified as "artifact" are not shown at all, although they can be accessed through the data search page (i.e., they are hidden but not deleted). This display significantly reduces the number of candidates to review and prioritizes those most likely to be of interest at the top of the page. We employed a two-week testing and evaluation period to assess whether this major change to candidate display was desirable and useful. The review team confirmed its utility, and this mode is now the default sort ordering.

We also determined that there were other desired sorting options. We added an option by which users can switch between sorting by classifier prediction (as above), SNR (strongest signals first), or by dispersion measure (highest DM first). All three modes have value.

\section{Results}

We have tracked and evaluated the impact of these improvements to the V-FASTR system in terms of reviewer time saved and a reviewer opinion survey.

\subsection{Reviewer Time Saved}

First, we conducted a retrospective evaluation of the classifier's performance and potential benefit using the data and candidates that were collected from January 2014 to February 2015. The classifier was not actually in use for filtering candidates during this period, but the data collected during that period allowed us to determine how it would have performed. Starting with January 2014, at the start of each month, we trained a classifier on all labeled candidates obtained prior to that month. We then used that classifier to generate blind predictions for new candidates observed during that month. Predictive accuracy started at $97 \%$ and quickly climbed to $99-100 \%$ (see Figure 8, blue dashed line).

We also measured the amount of reviewer effort that would be saved by using the classifier's predictions to filter out "artifact" and "known pulsar" candidates, so that the reviewers only needed to examine "good candidates" and unclassifiable candidates. The fraction of candidates confidently filtered by the classifier varied between 48-79\% from January to September of 2014 and then began to rise, reaching $89 \%$ by February 2015. This indicates that the posterior confidence estimates of the classifier improved as more labeled candidates were available for training, enabling the confident classification of more candidates. Ultimately, human reviewers needed only to examine $\sim 10 \%$ of the incoming candidates, providing a major time savings.

At the time of this writing, V-FASTR has collected 197,934 candidates. Of these, humans have labeled 4,632 pulsar pulses and 3,108 artifacts. We applied the classifier to the 102,147 candidates detected since January 1, 2014, from which it confidently labeled 20,096 pulsar pulses and 45,066 artifacts. The sky distribution of these candidates is shown in Figure 9. Note that these are the 


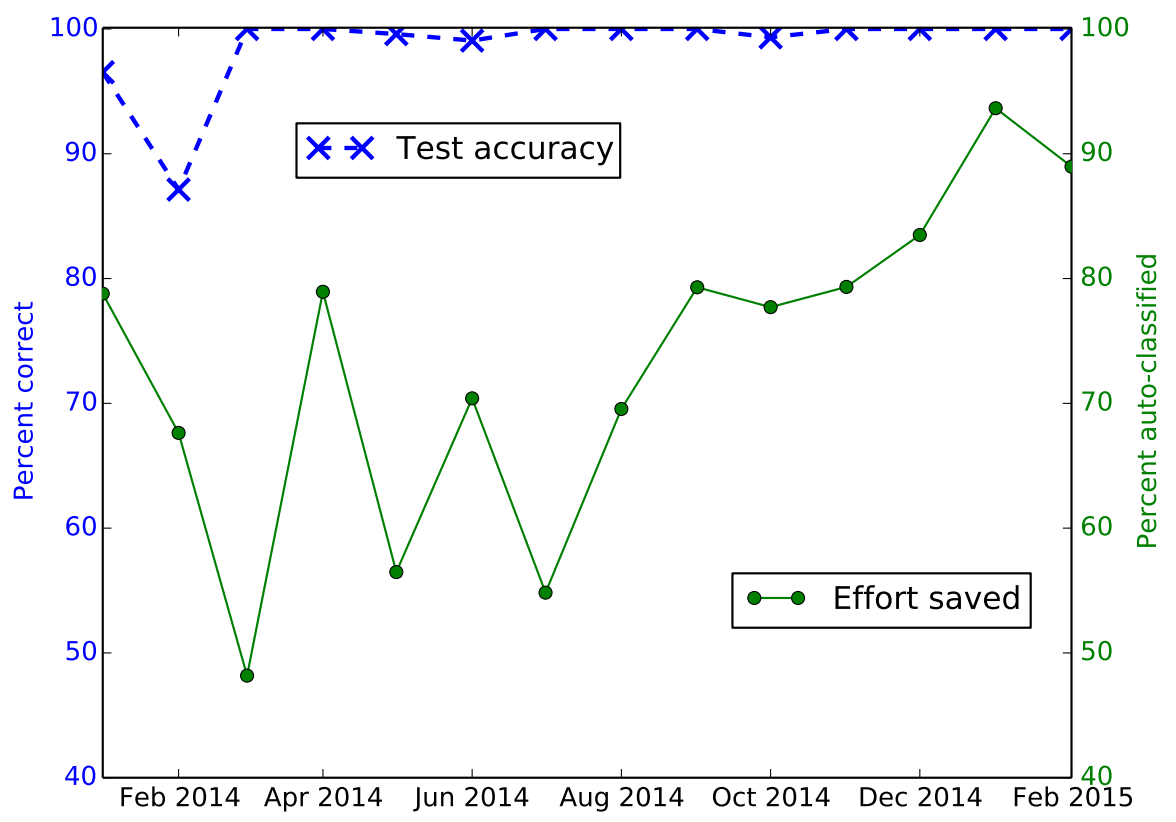

Fig. 8.- Retrospective study showing the candidate classifier performance as a function of time. For each month, we trained a classifier on all preceding labeled data. The accuracy of that classifier's blind predictions on new candidates observed that month is shown by the blue dashed line. The reviewer effort that would be saved by using the classifier's predictions to filter the candidates (auto-classify all "artifact" and "known pulsar" candidates) is shown in green.

locations of the array pointing center, not the candidates themselves. The pulsar pulses originate from three primary locations. VLBA PIs frequently use pulsars as calibration sources or as objects of study, so it is not surprising that the same pulsars would be re-visited by the array. In contrast, artifacts appear at diverse pointings all over the sky. There is a concentration of artifacts located along the galactic plane (diagonal line near RA 4.5-5.5, DEC -0.5-0.6) which is likely due to the popularity of this area as an observing location rather than any inherent increase in RFI at that pointing.

The V-FASTR classifier has also identified 28 good candidates, which have received special scrutiny. In each case so far, these candidates were determined to originate from a known pulsar location, but with an estimated DM that was quite different from the known value of the source pulsar. It is possible that these are FRBs from a source that is very near a known pulsar, but more likely that these particular pulses exhibit some variation or RFI corruption that causes the system to mis-estimate the DM value. However, these unusual events are precisely the ones that merit careful human attention, and the time spent evaluating them is therefore well spent. 


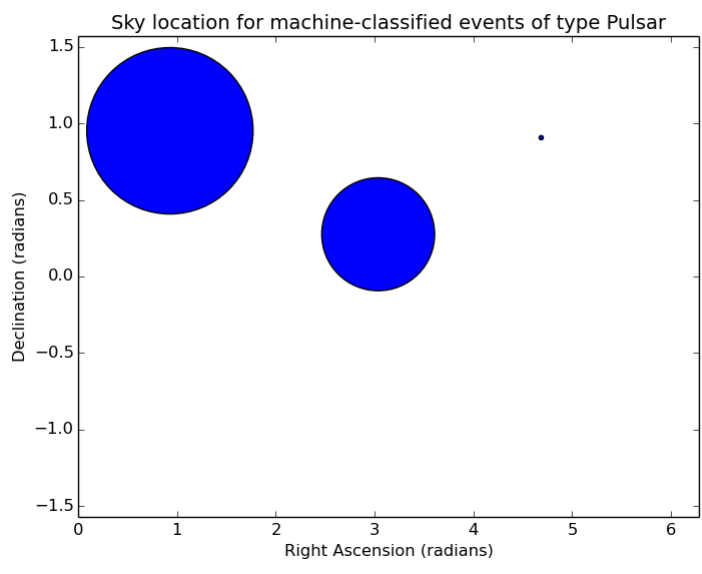

(a) Pulsar

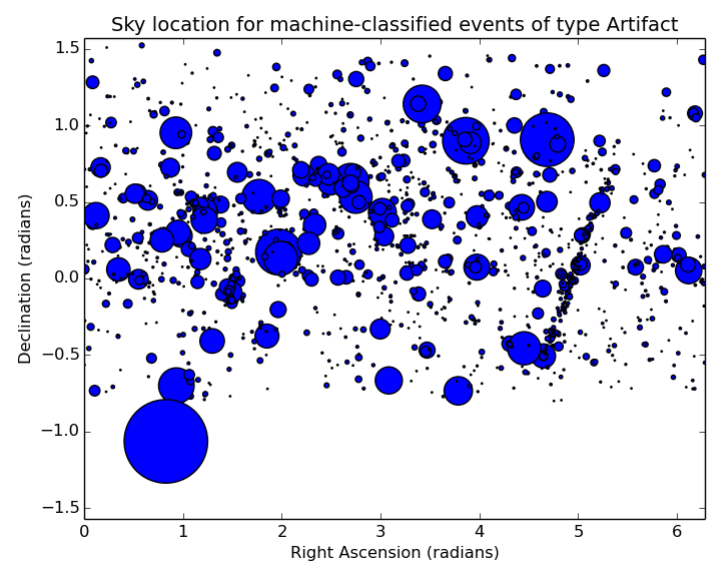

(b) Artifact

Fig. 9.- Sky location of candidates automatically classified as pulsar pulses or artifacts. The size of each circle is proportional to the number of automatically classified candidates found when the array was pointed at that sky location.

\subsection{Reviewer Survey Results}

The preceding results show that the V-FASTR classifier can greatly reduce reviewer effort while maintaining high reliability. We also conducted surveyed the reviewers to assess the utility of the V-FASTR classifier and web portal improvements from their perspective.

We conducted two surveys, one in September 2013 and the second in September 2015. The first survey established a baseline, using the original web portal, against which we could compare the later experiences after the improvements described in this paper were implemented. There were a total of six reviewers that participated in the surveys.

Our primary finding is that reviewers reported spending less time reviewing. The average self-reported time spent reviewing decreased from 32 to 16 minutes per week. As described above, the number of candidates presented to the reviewers has decreased greatly (to 10-20\% of the total number). However, this is somewhat conflated with a decrease in the total number of jobs going through the system since March 2015 due to a policy change.

We also found that the self-reported time spent per candidate increased, from an average of 5.4 to 10.2 seconds. This suggests that the candidates being reviewed require additional thought and are potentially more ambiguous or more interesting to the reviewer. When asked to report the percent of candidates that the reviewers considered (subjectively) interesting, the average increased slightly from $0.9 \%$ to $1.2 \%$. Because interesting candidates are rare, it is not surprising that this percentage is low. However, we aim to continue increasing the fraction.

Our most recent survey culminated in a question aimed specifically at assessing the impact 
of the incorporation of the classifier into the system. We asked: "Has the incorporation of the classifier, which now hides candidates tagged as 'pulsar' or 'artifact', reduced the time/effort you invest in reviewing?" As shown in the following table, we found that 4 of 6 reviewers reported benefiting from the classifier's decisions.

\begin{tabular}{|l|l|}
\hline Votes & Response \\
\hline 2 & Yes. I spend much less time (or effort) in reviewing each job. \\
2 & Yes. I spend a little less time (or effort) in reviewing each job. \\
0 & No. I spend about the same amount of time (or effort) in reviewing each \\
& job, compared to the previous system with no classifier-based filtering. \\
2 & I'm not sure; I haven't had enough recent jobs to review to see a difference. \\
\hline
\end{tabular}

\section{Conclusions and Future Work}

In this paper, we have presented two major improvements to the V-FASTR fast transient detection and review system. First, we incorporated a machine learning classifier to automatically filter out known types of events (pulsar pulses and artifacts) and enable human reviewers to devote their time to the most promising candidates. This classifier has a $98.6 \%$ accuracy on historical data and a 99-100\% accuracy on (labeled) newly collected data. It retains only predictions of at least 0.9 confidence. This reduces the reviewer workload by $80-90 \%$; only $\sim 10 \%$ of the candidates need human eyes. The remaining candidates can still be accessed through the V-FASTR archive, enabling the compilation of statistics in terms of candidate sky location, DM, SNR, etc.

Second, we implemented several efficiency and usability improvements to the V-FASTR web review portal that has streamlined the review process and made it possible for us to open the V-FASTR archive to the public.

These improvements have enhanced the ability of the V-FASTR science team to quickly review and tag candidate transient events detected during commensal processing of VLBA observations. A similar approach could be used in the future to manage the large volume of interesting candidates that are anticipated to be collected by the Square Kilometre Array and other future instruments.

Facilities: VLBA, NRAO.

We would like to thank Benyang Tang for his original version of the V-FASTR candidate classifier, Luca Cinquini and Andrew Hart for the initial web portal and data crawler architecture design and implementation, Tyler Palsulich for integrating the pulsar database into web portal, and Sarah Burke-Spolaor, Walter Brisken, and Walid Majid for contributing labeled data and feedback on the classifier and portal system.

The International Center for Radio Astronomy Research is a joint venture between Curtin University and The University of Western Australia, funded by the State Government of Western 
Australia and the joint venture partners. Steven J. Tingay is a Western Australian Premiers Research Fellow. This work was done in part at the Jet Propulsion Laboratory under a Research and Technology Development Grant, under contract with the National Aeronautics and Space Administration. Copyright 2015. All Rights Reserved. US Government Support Acknowledged. 


\section{REFERENCES}

Breiman, L. (2001). Random forests. Machine Learning, 45:5-32.

Burke-Spolaor, S., Bailes, M., Ekers, R., Macquart, J.-P., and Crawford III, F. (2011). Radio bursts with extragalactic spectral characteristics show terrestrial origins. The Astrophysical Journal, 727(1):18.

Burke-Spolaor, S. and Bannister, K. W. (2014). The galactic position dependence of fast radio bursts and the discovery of FRB011025. The Astrophysical Journal, 792(19).

Deller, A. T., Brisken, W. F., Phillips, C. J., Morgan, J., Alef, W., Cappallo, R., Middelberg, E., Romney, J., Rottmann, H., Tingay, S. J., and Wayth, R. (2011). DiFX-2: A more flexible, efficient, robust, and powerful software correlator. Proceedings of the Astronomical Society of the Pacific, 123:275-287.

Dewdney, P. E., Hall, P. J., Schilizzi, R. T., and Lazio, T. (2009). The Square Kilometre Array. Proceedings of the IEEE, 97(8):1482-1496.

Falcke, H. and Rezzolla, L. (2014). Fast radio bursts: The last sign of supramassive neutron stars. Astronomy \& Astrophysics, 562.

Fender, R., Stewart, A., Macquart, J. P., Donnarumma, I., Murphy, T., Deller, A., Paragi, Z., and Chatterjee, S. (2015). The transient universe with the Square Kilometre Array. In Proceedings of Advancing Astrophysics with the Square Kilometre Array.

Hart, A. F., Cinquini, L., Khudikyan, S. E., Thompson, D. R., Mattmann, C. a., Wagstaff, K., Lazio, J., and Jones, D. (2014). A framework for collaborative review of candidate events in high data rate streams: The V-FASTR experiment as a case study. The Astronomical Journal, 149(1):23.

Kashiyama, K., Ioka, K., and Mészáros, P. (2013). Cosmological fast radio bursts from binary white dwarf mergers. The Astrophysical Journal Letters, 776(2).

Keane, E. F., Stappers, B. W., Kramer, M., and Lyne, A. G. (2012). On the origin of a highly dispersed coherent radio burst. Monthly Notices of the Royal Astronomical Society, 425:L71L75.

Law, C. J., Bower, G. C., Burke-Spolaor, S., Butler, B., Lawrence, E., Lazio, T. J. W., Mattmann, C. A., Rupen, M., Siemion, A., and VanderWiel, S. (2015). A millisecond interferometric search for fast radio bursts with the Very Large Array. The Astrophysical Journal, 807(1).

Loeb, A., Shvartzvald, Y., and Maoz, D. (2014). Fast radio bursts may originate from nearby flaring stars. Monthly Notices of the Royal Astronomical Society: Letters, 439(1):45-50. 
Lorimer, D. R., Bailes, M., McLaughlin, M. A., Narkevic, D. J., and Crawford, F. (2007). A bright millisecond radio burst of extragalactic origin. Science, 318(5851):777-780.

Macquart, J. P., Keane, E., Grainge, K., McQuinn, M., Fender, R., Hessels, J., Deller, A., Bhat, R., Breton, R., Chatterjee, S., Law, C., Lorimer, D., Ofek, E. O., Pietka, M., Spitler, L., Stappers, B., and Trott, C. (2014). Fast transients at cosmological distances with the SKA. In Proceedings of Advancing Astrophysics with the Square Kilometre Array.

Manchester, R. N., Hobbs, G. B., Teoh, A., and Hobbs, M. (2005). The ATNF pulsar catalogue. The Astronomical Journal, 129. http://www.atnf.csiro.au/research/pulsar/psrcat.

Masui, K., Lin, H.-H., Sievers, J., Anderson, C. J., Chang, T.-C., Chen, X., Ganguly, A., Jarvis, M., Kuo, C.-Y., Li, Y.-C., Liao, Y.-W., McLaughlin, M., Pen, U.-L., Peterson, J. B., Roman, A., Timbie, P. T., Voytek, T., and Yadav, J. K. (2015). Dense magnetized plasma associated with a fast radio burst. Nature, 528:523-525.

Mattmann, C., Hart, A., Cinquini, L., Lazio, J., Khudikyan, S., Jones, D., Preston, R., Bennett, T., Butler, B., Harland, D., Cummings, K., Glendenning, B., Kern, J., and Robnett, J. (2013). Big Data Management, Technologies, and Applications, chapter Scalable Data Mining, Archiving and Big Data Management for the Next Generation Astronomical Telescopes, pages 196-221. IGI Global.

Petroff, E., Bailes, M., Barr, E. D. D., Barsdell, B. R. R., Bhat, N. D. R. D. R., Bian, F., BurkeSpolaor, S., Caleb, M., Champion, D., Chandra, P., Da Costa, G., Delvaux, C., Flynn, C., Gehrels, N., Greiner, J., Jameson, A., Johnston, S., Kasliwal, M. M. M., Keane, E. F. F., Keller, S., Kocz, J., Kramer, M., Leloudas, G., Malesani, D., Mulchaey, J. S. S., Ng, C., Ofek, E. O. O., Perley, D. A. A., Possenti, A., Schmidt, B. P. P., Shen, Y., Stappers, B., Tisserand, P., van Straten, W., and Wolf, C. (2015a). A real-time fast radio burst: Polarization detection and multiwavelength follow-up. Monthly Notices of the Royal Astronomical Society, $447(1): 246-255$.

Petroff, E., Keane, E. F., Barr, E. D., Reynolds, J. E., Sarkissian, J., Edwards, P. G., Stevens, J., Brem, C., Jameson, A., Burke-Spolaor, S., Johnston, S., Bhat, N. D. R., Chandra, P., Kudale, S., and Bhandari, S. (2015b). Identifying the source of perytons at the Parkes radio telescope. Monthly Notices of the Royal Astronomical Society, 451(4):3933-3940.

Ravi, V., Shannon, R. M., and Jameson, A. (2015). A fast radio burst in the direction of the Carina Dwarf Spheroidal galaxy. The Astrophysical Journal Letters, 799:L5.

Siemion, A. P. V., Bower, G. C., Foster, G., McMahon, P. L., Wagner, M. I., Werthimer, D., Backer, D., Cordes, J., and van Leeuwen, J. (2012). The Allen Telescope Array Fly's Eye Survey for fast radio transients. The Astrophysical Journal, 744(2):109.

Spitler, L. G., Cordes, J. M., Hessels, J. W. T., Lorimer, D. R., McLaughlin, M. A., Chatterjee, S., Crawford, F., Deneva, J. S., Kaspi, V. M., Wharton, R. S., Allen, B., Bogdanov, S., 
Brazier, A., Camilo, F., Freire, P. C. C., Jenet, F. A., Karako-Argaman, C., Knispel, B., Lazarus, P., Lee, K. J., van Leeuwen, J., Lynch, R., Ransom, S. M., Scholz, P., Siemens, X., Stairs, I. H., Stovall, K., Swiggum, J. K., Venkataraman, A., Zhu, W. W., Aulbert, C., and Fehrmann, H. (2014). Fast radio burst discovered in the Arecibo Pulsar ALFA Survey. The Astrophysical Journal, 790(2):101.

Thompson, D. R., Brisken, W. F., Deller, A. T., Majid, W. A., Burke-Spolaor, S., Tingay, S. J., Wagstaff, K. L., and Wayth, R. B. (2013). Real time adaptive event detection in astronomical data streams: Lessons from the Very Long Baseline Array. IEEE Intelligent Systems, 29(1):48-55.

Thompson, D. R., Wagstaff, K. L., Brisken, W., Deller, A. T., Majid, W. a., Tingay, S. J., and Wayth, R. B. (2011). Detection of fast radio transients with multiple stations: A case study using the Very Long Baseline Array. The Astrophysical Journal, 98:12.

Thornton, D., Stappers, B., Bailes, M., Barsdell, B., Bates, S., Bhat, N. D. R., Burgay, M., BurkeSpolaor, S., Champion, D. J., Coster, P., D’Amico, N., Jameson, A., Johnston, S., Keith, M., Kramer, M., Levin, L., Milia, S., Ng, C., Possenti, A., and van Straten, W. (2013). A population of fast radio bursts at cosmological distances. Science, 341:53-56.

Totani, T. (2013). Cosmological fast radio bursts from binary neutron star mergers. Publications of the Astronomical Society of Japan, 65(5).

Trott, C. M., Tingay, S. J., Wayth, R. B., Thompson, D. R., Deller, A. T., Brisken, W. F., Wagstaff, K. L., Majid, W. A., Burke-Spolaor, S., Macquart, J.-P. R., and Palaniswamy, D. (2013). A framework for interpreting fast radio transients search experiments: Application to the V-FASTR experiment. The Astrophysical Journal, 767(4).

Wayth, R. B., Brisken, W. F., Deller, A. T., Majid, W. a., Thompson, D. R., Tingay, S. J., and Wagstaff, K. L. (2011). V-fastr: The VLBA fast radio transients experiment. The Astrophysical Journal, 97(2007):14.

Wayth, R. B., Tingay, S. J., Deller, A. T., Brisken, W. F., Thompson, D. R., Wagstaff, K. L., and Majid, W. a. (2012). Limits on the event rates of fast radio transients from the V-FASTR experiment. The Astrophysical Journal, 753(2007):L36.

Zhang, B. (2014). A possible connection between fast radio bursts and gamma-ray bursts. The Astrophysical Journal Letters, 780(2). 